\title{
Cognitive performance and informant reports in the diagnosis of cognitive impairment and dementia in African Americans and whites
}

\author{
Guy G. Potter ${ }^{\mathrm{a}, *}$, Brenda L. Plassman ${ }^{\mathrm{a}}$, James R. Burke ${ }^{\mathrm{b}}$, Mohammed U. Kabeto ${ }^{\mathrm{c}}$, \\ Kenneth M. Langa ${ }^{\mathrm{c}, \mathrm{d}, \mathrm{e}}$, David J. Llewellyn ${ }^{\mathrm{f}}$, Mary A. M. Rogers ${ }^{\mathrm{d}}$, David C. Steffens ${ }^{\mathrm{a}}$ \\ ${ }^{a}$ Department of Psychiatry and Behavioral Sciences, Duke University Medical Center, Durham, NC, USA \\ ${ }^{b}$ Division of Neurology, Department of Medicine, Duke University Medical Center, Durham, NC, USA \\ ${ }^{c}$ Institute for Social Research, University of Michigan, Ann Arbor, MI, USA \\ ${ }^{d}$ Division of General Medicine, Department of Medicine, University of Michigan, Ann Arbor, MI, USA \\ ${ }^{e}$ Veterans Affairs Center for Practice Management and Outcomes Research, Ann Arbor, MI, USA \\ ${ }^{f}$ Institute of Public Health, University of Cambridge, Cambridge, United Kingdom
}

\begin{abstract}
Background: The diagnosis of cognitive impairment and dementia must reflect an increasingly diverse and aging United States population. This study compared direct testing and informant reports of cognition with clinical diagnoses of cognitive impairment and dementia between African Americans and whites.

Methods: Participants in the Aging, Demographics, and Memory Study completed in-person dementia evaluations, and were assigned clinical diagnoses (by a consensus panel of dementia experts) of normal; cognitive impairment, not demented (CIND); and dementia. The Consortium to Establish a Registry for Alzheimer's Disease (CERAD) total score and the Informant Questionnaire on Cognitive Decline in the Elderly (IQCODE) were used to assess cognitive performance and reported cognitive decline.

Results: A higher CERAD total score was associated with lower odds of CIND and dementia, at comparable ratios between African Americans and whites. Higher IQCODE scores were associated with increased odds of dementia in both African Americans and whites. Higher IQCODE scores were associated with increased odds of CIND among whites, but not among African Americans.

Conclusions: Cultural differences may influence informant reports of prevalent CIND and dementia. Our findings also highlight the need for more comparative research to establish the cultural validity of measures used to diagnose these conditions.

(C) 2009 The Alzheimer's Association. All rights reserved.
\end{abstract}

\section{Introduction}

The growing proportion of older adults in the United States population makes for a growing public-health concern about age-related cognitive impairment and dementia. Adults aged over 65 years are estimated to make up $20 \%$ of the United States population in 2050 . However, within this demographic trend, there is an estimated decline in the proportion of (non-Hispanic) white Americans, and estimated increases in proportions

*Corresponding author. Tel.: 919-682-2722; Fax: 919-687-0424.

E-mail address: guy.potter@duke.edu of African Americans, Latino-Hispanic Americans, and AsianPacific Americans [1]. To date, most studies of racial and ethnic differences in cognitive impairment and dementia in the United States have focused on differences between African Americans and whites, and some found prevalence rates of Alzheimer's disease $(\mathrm{AD})$ and dementia to be approximately twice as high among African Americans [2,3]. Although these differences are sometimes present when controlling for demographic, sociocultural, and health-related variables [2], other studies found the higher rate of dementia among African Americans to be explained in part by differences in age, education, and genetic susceptibility [4,5]. Less is known about racial and ethnic 
differences at milder levels of cognitive impairment, where interventions may be more effective in slowing or preventing the conversion to dementia. The one population-based study of cognitive impairment making an explicit comparison of prevalence rates in African Americans and whites found African Americans to have a 4.4 times greater risk of mild cognitive impairment (MCI) than whites [6]. However, the authors also noted that racial and ethnic differences in apolipoprotein $E$ (APOE) $\varepsilon 4$ status and cardiovascular health may underlie much of this disparity. On the whole, studies of the prevalence of cognitive impairment and dementia among African Americans have found comparable-to-higher rates relative to whites, but much of this variability may be attributable to underlying socioeconomic and cultural differences in education, geography, diagnostic methodology, and other factors. Projecting forward, the effectiveness of the public-health response to the expected increase in age-related cognitive impairment and dementia may be closely linked to effectiveness in detecting and diagnosing these conditions in a population of increasing cultural diversity.

Although a diagnosis of cognitive impairment and dementia in clinical and epidemiological studies of diverse populations should ideally result from multiple sources of information, including clinical and medical history, social history, demographic characteristics, and cognitive and functional assessments, two of the core sources of information consist of direct assessments of cognitive performance, and informant reports of cognitive ability. One concern about a direct cognitive assessment involves its possible contribution to a higher rate of diagnosed cognitive impairment among African Americans, because the influence on test performance of ethnic differences in such factors as educational quality and acculturation is not well-explained at present [7]. Studies showed that cutoffs based on brief cognitive screening tests can produce higher rates of dementia and MCI among African Americans, even when controlling for level of education [8,9]. Neuropsychological test batteries designed for sensitivity to dementia also demonstrate lower performances among African Americans relative to whites, even when matching for education, other socioeconomic variables, and medical comorbidities [10-12]. Disparities in cognitive performance between African Americans and whites may reduce the specificity of these measures and increase the risk of overdiagnosing cognitive impairment among African Americans, particularly when these cognitive changes are mild. Separate norms may improve specificity in the detection of cognitive impairment, but they may not address underlying educational and cultural differences that could also influence diagnostic accuracy [7].

Informant-based reports provide another source of information for diagnosing cognitive impairment and dementia, but less is known about whether perceptions of cognitive function are influenced by ethnic differences. On the one hand, informant-based assessments tend to implicitly or explicitly require a judgment of the subject's cognitive and functional ability relative to a previous level of performance, which, unlike direct cognitive testing, may be less influenced by a subject's educational or cultural background. On the other hand, educational and cultural factors may differentially influence an informant's perception of the nature and severity of cognitive difficulties. One study in an AD sample found that African American family caregivers overrated the subject's cognitive function, whereas white family caregivers underrated it [13]. However, different results may exist across a broader range of cognitive ability, and outside of the caregiver context. With respect to specific measures of informant reports, the Informant Questionnaire on Cognitive Decline in the Elderly (IQCODE) is one of the most widely used informant measures of cognitive decline, and has demonstrated utility in detecting dementia in multiple cultural groups, including Australian, Spanish, and Chinese samples [14-16]. In a study using a Singaporean sample, for instance, the combination of cognitive testing and IQCODE provided a better detection of dementia when individuals had no education [17]. A study using a Spanish sample found that IQCODE was more sensitive to mild dementia than the Mini-Mental State Examination (MMSE), after controlling for effects of age, gender, and education [15]. Although IQCODE is widely used to diagnose cognitive impairment, we are aware of no studies that examined the validity of IQCODE for detecting cognitive impairment among African Americans. Moreover, there has been little research overall on how IQCODE is associated with normal cognition, cognitive impairment (without dementia), and dementia in the same sample.

The goal of this study was to compare the relative associations of cognitive testing and IQCODE to diagnoses of cognitive impairment and dementia between African Americans and whites. We examined not only the prediction of dementia, but also the cognitive impairment that exists in the absence of a formal diagnosis of dementia, often characterized as "cognitive impairment, no dementia" (CIND). Clinically, this designation may include either subjective or objective reports of mild cognitive or functional impairment, based on interviews or formal assessment. We hypothesized that informant reports would be less likely to predict CIND among African Americans than direct cognitive testing. On the one hand, the presentation of CIND is often less overt and newly emergent, which can lead to an underdiagnosis of subtle cognitive changes. On the other hand, ethnic disparities in test performance may lead to an overdiagnosis of subtle cognitive impairment. We examined this question in the context of the Aging, Demographics, and Memory Study (ADAMS), which was designed to provide nationally representative data on the antecedents, prevalence, and outcomes of cognitive impairment and dementia in the United States.

\section{Methods}

\subsection{Participants}

The ADAMS sample was drawn from the larger Health and Retirement Study (HRS), which is an ongoing, nationally representative cohort study of individuals born before 1954, designed to investigate the health, social, and economic implications of aging in the American population [18-20]. 
The HRS began in 1992, and the current sample includes approximately 22,000 participants.

The ADAMS sample began with a stratified random subsample of 1770 individuals aged $\geq 70$ years from five cognitive strata, defined according to scores on a cognitive measure completed by the participant, or a proxy-reported cognitive measure [21] from the most recent HRS interview (either 2000 or 2002). The three highest cognitive strata were further stratified by age ( $70-79$ years vs. $\geq 80$ years) and sex, to ensure adequate numbers in each subgroup. Full details of the ADAMS sample design and selection procedures are described elsewhere [22,23]. The initial ADAMS assessments occurred between July 2001 and December 2003, on average, 13.3 (SD, 6.9) months after the HRS interview. Thus, participants were aged $\geq 71$ years at the time of initial assessment. Racial and ethnic identification was obtained by self-report, based on United States Census categories. The ADAMS sample of 856 represents $56 \%$ of the surviving targeted sample. Of 856 ADAMS participants, 645 (75.4\%) were used in the final analyses. This number resulted from exclusion criteria related to incomplete informant reports or cognitive testing that could not be completed because of impairments in vision, hearing, or motor function. In addition, participants who did not indicate race/ethnicity as African American or white were excluded for the purposes of the present study $(n=34)$.

As a population-representative study, ADAMS had to account for nonparticipation rates and sample methodology. Covariates of age, gender, education, marital status, HRS cognition scores, nursing-home residency, and indicators of previous or existing major health conditions were used in a response propensity analysis, to develop nonresponse adjustments to the ADAMS sample selection weights [24]. Population sample weights were then constructed to take into account the probabilities of selection in the stratified sample design, and to adjust for differential nonparticipation in ADAMS [22]. These values were weighted back to the United States population. The ADAMS data are publicly available, and can be obtained from the HRS website [25]. The Institutional Review Boards at Duke University Medical Center and the University of Michigan approved all study procedures, and informed consent was provided by study participants or their surrogates.

\subsection{Dementia assessment and diagnosis}

All participants were assessed for cognitive impairment in-person at their residence by a nurse and a neuropsychology technician. The full details of the assessment and diagnostic procedures were previously described [23]. During the assessment, the participant completed: 1) a battery of neuropsychological measures, 2) a self-report depression measure, 3 ) a standardized neurological examination, 4) a blood-pressure measure, 5) a collection of buccal DNA samples for $A P O E$ genotyping, and 6) a 7-minute videotaped segment containing portions of the cognitive status and neurological examinations. A knowledgeable informant provided the following information about each participant: 1) a chronological history of cognitive and functional symptoms, 2) a medical history, 3) current medications, 4) current neuropsychiatric symptoms, 5) measures of severity of cognitive and functional impairment, and 6) a family history of memory problems. The informant was usually a spouse or child (73\%), and informants lived with the participant in just over half of the cases (54\%); there were no differences between African Americans and whites in either regard. Medical-record releases were also requested, to obtain relevant neuroimaging and laboratory results from participants' physicians.

Clinical diagnoses in ADAMS were assigned by a consensus expert panel of neuropsychologists, neurologists, geropsychiatrists, and internists. Final diagnoses for each participant were assigned after the diagnostic panel reviewed all available information from the in-home assessment and any available medical records or neuroimaging results. Although IQCODE was completed in association with the study visit, its score was not presented for clinical consideration. The assessment and diagnostic methods described in the present study have: 1) been used in numerous large epidemiological studies of dementia (the Duke Twins Study, the Veterans Study of Memory in Aging, and the Cache County Memory Study) [26-28]; 2) demonstrated agreement rates for a clinical and pathological diagnosis of $\mathrm{AD}$ that are comparable to those in tertiary-care centers [29]; and 3) found an acceptable agreement rate for an $\mathrm{AD}$ diagnosis, based on nurse-led assessment and later diagnosis of the same patient after physician-led assessment [29].

Diagnoses fell within three general categories: normal cognitive function, CIND, and dementia. Although the consensus panel used clinical judgment to assign final diagnoses, each diagnosis was anchored by published clinical criteria and guidelines. A diagnosis of dementia was based on guidelines from the Diagnostic and Statistical Manual of Mental Disorders, Edition III-R [30], and criteria from the Diagnostic and Statistical Manual of Mental Disorders, Edition IV [31]. Currently accepted diagnostic criteria for $\mathrm{AD}$ and other types of dementia were also used [32-35]. The operational definitions for CIND and its subtypes were developed over a 17-year period, primarily based on the accumulated clinical experience of a group of researchers common to ADAMS and three other epidemiologic studies of dementia $[26,27,36]$. We defined CIND as: 1) mild cognitive or functional impairment, as reported by a participant or informant, that did not meet the criteria for dementia; or 2) a performance on neuropsychological measures that was both below expectations and $\geq 1.5$ SDs below the published norms on any test. Individual subcategories of CIND are described elsewhere [37].

\subsection{Measures}

\subsubsection{Consortium to Establish a Registry for Alzheimer's Disease total score}

Cognitive performance was assessed by a total score of tests from the Consortium to Establish a Registry for 
Alzheimer's Disease (CERAD) neuropsychological battery [38]. The CERAD total score (TS) was found to be highly accurate in differentiating independent samples of white participants with normal cognitive function, MCI, and AD. In addition, the CERAD TS was found to be highly correlated with the MMSE $(r=0.89)$ and Clinical Dementia Rating Scale $(r=0.83)$ in a sample of individuals with normal cognition and AD [39]. The CERAD battery includes Animal Naming, the 15-item Boston Naming Test, Constructional Praxis, and a Word List Memory (learning, delayed recall, and recognition). The MMSE is typically part of the CERAD battery, but is not included in the CERAD TS. Although several other neuropsychological measures were part of the ADAMS clinical assessment, the CERAD TS produces a single score that offers the utility of a broader and more detailed assessment of cognitive function than the MMSE, while avoiding floor effects among a sample including dementia.

\subsubsection{IQCODE}

The IQCODE was developed to measure cognitive decline from a previous level (typically 10 years earlier), based on informant report $[14,40]$. Our study used the 16 -item version, which was reported to be essentially comparable to the full version [41]. The version in the present study asked informants to rate cognitive and functional change over the past 2 years. Cognitive and functional change on IQCODE is rated on a 5-point scale, ranging from 1 to 5, i.e., Much better, A bit better, Not much change, A bit worse, and Much worse.

\subsection{Statistical methods}

Statistical analyses were performed using Stata 9.1 [42]. Univariate statistics were used to inspect means and distributions of variables. Pearson's product-moment correlations were used to examine associations between CERAD TS and IQCODE. We used $t$-tests and $\chi^{2}$ tests to examine group differences across variables. We used multinomial logistic regression to examine cognitive diagnosis as a function of CERAD TS and IQCODE score, while covarying for age, education, and gender. We coded our dependent variable (diagnosis) as an ordinal outcome (1, normal; 2, CIND; or 3 , demented). Although exponentiated coefficients in binomial logistic regression are odds ratios, the exponentiated coefficients in multinomial logistic regression are not calculated as ratios of odds, but rather as ratios of relative probabilities. Thus the model yields the ratio of the relative probability for a one-unit increase in $x$ to the relative probability when $x$ is unchanged. In Stata, models were fitted using the "mlogit" command [43]. Because probabilities are often transformed to odds, the exponentiated coefficients from multinomial logistic regression can also be called "conditional odds ratios."

Although the scaling of IQCODE produces a possible score range of 1 to 5 , much of the clinical sensitivity of this measure lies in the gradation between mean scores of 3 (Not much change) and 4 (A bit worse). To improve the interpretability of changes in IQCODE scores on the odds of diagnostic outcomes, we rescaled the original observed minimum $(\mathrm{IQCODE}=1)$ and maximum $(\mathrm{IQCODE}=5)$ scores to a range of 0 to 100 , based on the following formula: $100 \times(\mathrm{IQCODE}-$ minimum IQCODE)/(maximum IQCODE - minimum IQCODE). In rescaled form, a unit of change in the odds ratio corresponded to a change of 0.04 on the original IQCODE scale. All analyses were weighted, using the complex sampling design of ADAMS. Because separate analyses for African Americans and whites were performed according to the CERAD TS (see below), a subpopulation analysis was used to obtain the correct standard errors for estimates.

\section{Results}

As described previously (in "2.1. Participants"), 645 of 856 ADAMS respondents were used in the final analyses. Of these 645 respondents, 111 (design-weighted, 6.9\%) were African American. Among African Americans, 32 were diagnosed as normal (design-weighted, 52.2\%), 35 were diagnosed with CIND (design-weighted, 26.5\%), and 35 were diagnosed with dementia (design-weighted, 21.3\%). Among whites, 230 were diagnosed as normal (design-weighted, 68.1\%), 142 were diagnosed with CIND (design-weighted, 20.8\%), and 162 were diagnosed with dementia (design-weighted, $11.2 \%)$. Design-based Pearson $\chi^{2}$ indicated a significant association between racial group and diagnosis $(P=0.046)$. Our initial inspection of distributions of CERAD TS by race indicated approximately parallel slopes for the cognitive performance of African Americans and whites as a function of clinical diagnosis (Fig. 1). This presented a confound in examining the association of cognitive performance with diagnosis among all subjects, so we conducted separate multinomial logistic models for African Americans and whites.

Table 1 shows the demographic characteristics of the sample, stratified by race and diagnosis. There were no differences between African Americans and whites in age or gender, either overall or across diagnoses. African Americans did have a lower level of education overall, and across each diagnostic group. Means and standard errors of the CERAD TS and IQCODE also appear in Table 1, and indicated that the mean CERAD TS was lower among African Americans than whites, both overall and across each diagnostic group. There were no significant differences in mean IQCODE score between whites and African Americans for diagnoses of normal function and dementia. However, the mean IQCODE score was significantly higher (i.e., indicating more impairment) for whites than African Americans among individuals with a diagnosis of CIND $(P=0.02)$. The zero-order correlation between CERAD TS and IQCODE was significant among both African Americans $(r=-0.46, P=0.002)$ and whites $(r=-0.37, P<0.001)$ with a diagnosis of dementia, but there were no significant correlations between these measures for individuals diagnosed as normal and CIND. Fig. 1 shows the slopes of the CERAD TS stratified by race and diagnosis, and Fig. 2 shows the slopes of IQCODE stratified by race and diagnosis. 


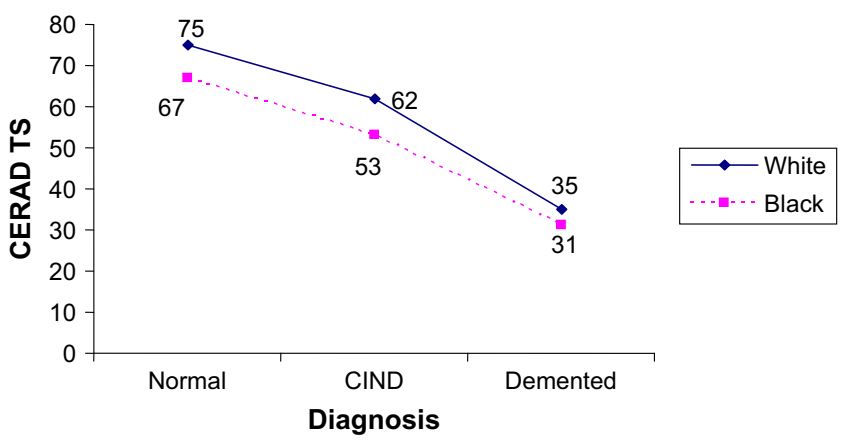

Fig. 1. Weighted means of CERAD TS by diagnosis and race.

Table 2 shows the association of CERAD TS with the odds of CIND and dementia among African American and white participants, covarying for age, gender, and education. The CERAD TS was the only significant predictor in models with African Americans, indicating that a higher CERAD TS was associated with lower odds of clinically diagnosed dementia and CIND. Among whites, a higher CERAD TS was also associated with lower odds of clinically diagnosed dementia and CIND. A higher level of education was associated with higher odds of dementia among whites when accounting for CERAD TS and other demographic factors, but it was not associated with a diagnosis of CIND. Because we found a significant association between education and CERAD TS in preliminary analyses, we repeated the regression models shown in Table 2 in a sensitivity analysis where only age, sex, and CERAD TS were included as predictors. The results did not change appreciably.

Table 3 shows the association of IQCODE with the odds of CIND and dementia among African American and white participants, covarying for age, gender, and education. None of the variables were significantly associated with a probability of CIND among African Americans, including IQCODE. However, IQCODE was the only variable associated with dementia, and a higher IQCODE score was associated with increased odds of dementia. The results for whites indicated that a higher IQCODE score was associated with increased odds of both CIND and dementia. In addition, higher levels of education were associated with lower odds of both CIND and dementia among white participants, but there was no association with education among African Americans.

\section{Discussion}

The present study examined associations of cognitive performance and informant reports of cognitive decline with the probabilities of CIND and dementia among African Americans and whites. We found that a better cognitive performance, as assessed by CERAD TS, was associated with lower odds of CIND and dementia among both African American and white participants, with similar odds ratios between groups for each of these diagnoses. We also found that informant reports of greater cognitive decline on IQCODE were associated with higher prevalent dementia odds among both African Americans and whites. For a diagnosis of CIND, however, greater informant reports of cognitive decline were associated with increased odds of this condition among whites, but not African Americans. These results suggest that informant reports of cognitive decline, as assessed by IQCODE, may differ between African Americans and whites for the detection of the milder cognitive and functional impairments of CIND. These results were independent of the covaried effects of age, education, and gender.

The mean differences in CERAD TS between African Americans and whites are consistent with previous studies finding that African Americans generally have lower scores on neuropsychological measures used in the assessment of cognitive impairment and dementia [12], including some tests within the CERAD battery [10]. In many cases, the evidence demonstrates that racial differences in cognitive performance are strongly associated with educational attainment, literacy, and quality of education. However, it may also be the case that educational variables are a proxy for acculturation and broader sociocultural factors [7]. Although racial and ethnic differences in neuropsychological test performances may raise concerns about diagnostic disparities, the slopes of mean CERAD TS scores were similar in each diagnostic category between African Americans and whites. Moreover, we found that the relative risks of CIND and dementia associated with CERAD TS performance were comparable among blacks and whites, when controlling for the effects of education, age, and gender.

With respect to our finding that IQCODE was associated with a diagnosis of CIND among whites but not African Americans, our results suggest that: 1) African American informants may be less inclined to report mild cognitive changes, 2) whites may be more inclined to report mild cognitive changes, or 3) both conditions may exist. Although there is limited research on how African American and white informants differ in perceiving cognitive changes as a function of magnitude or diagnosis, one study did find that African American caregivers overrated the care recipient's cognitive function, whereas white caregivers underrated cognitive ability [13]. Although most participants in the present study were independent and community-dwelling, it may be possible to compare our findings with those in the dementia caregiver literature, where the majority of studies found that African American caregivers reported less depression [44,45], more positive appraisal [46], less stress over behavioral disturbances $[44,47,48]$, and a lower caregiver burden [49] than did white dementia caregivers. Along with the present findings using IQCODE, this research suggests that cultural attitudes toward cognitive impairment and caregiving among African Americans and whites may produce different informant reports of cognitive decline. Previous studies of IQCODE in multiple countries suggested limited cultural differences in IQCODE, but those studies typically evaluated dementia within relatively homogenous cultural groups in their respective samples. We are not aware of a similar 
Table 1

Participant characteristics, cognitive performance, and informant reports of cognitive decline according to race and diagnosis $(\mathrm{n}=645)$



Abbreviations: CERAD TS, Consortium to Establish a Registry for Alzheimer's Disease neuropsychological battery, total score; IQCODE, Informant Questionnaire on Cognitive Decline in the Elderly; CIND, cognitive impairment, not demented; Educ, education level; Prob $\left(H_{0}\right)$, null hypothesis that group differences are not significant.

comparison of two cultural groups based on IQCODE for both cognitive impairment and dementia within the same sample, as was the case in the present study. More research is needed on IQCODE and other informant reports of cognitive decline in samples that allow for comparison of multiple cultural groups and different levels of cognitive decline. This issue is also relevant in other countries where differences in education, acculturation, and other factors may influence perspectives on cognitive change in the elderly.

We believe the current results have implications for the detection of CIND and dementia in clinical contexts, and for cognitive screening in population-based research. Although some normative values specific to African Americans exist for the CERAD battery [10,11], they do not exist for the CERAD TS, and there is a general lack of screening instru-

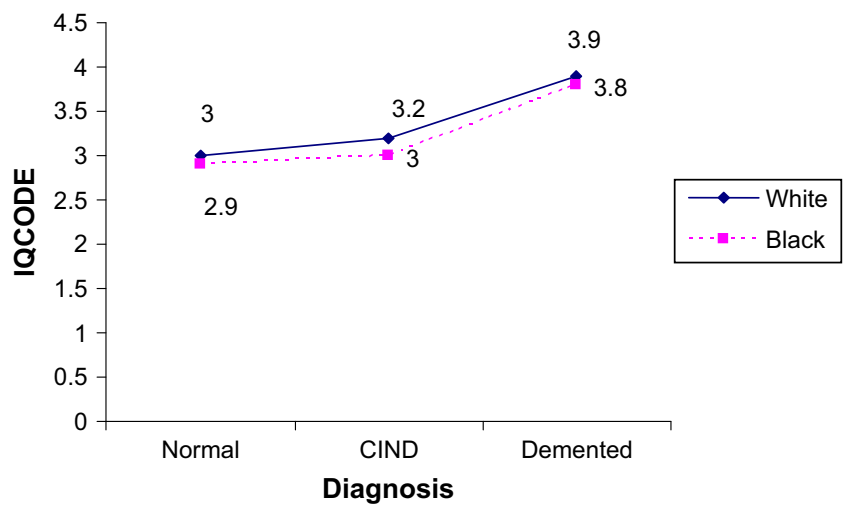

Fig. 2. Weighted means of IQCODE by diagnosis and race. ments for dementia and cognitive impairment from which appropriate cutoffs could be established with respect to age, education, and ethnicity. Informant reports such as IQCODE can potentially improve the community-based screening of cognitive impairment, because they are timeefficient and useful when cognitive testing is not feasible, and positive screening may be used to identify the need for additional neuropsychological evaluation. Similarly, although our results suggest that IQCODE may be useful in screening for dementia among both African Americans and whites, an independent sample is needed to establish appropriate values for sensitivity and specificity in each cultural group. In the case of cognitive impairment in the absence of dementia, our results suggest that IQCODE may be useful in identifying cognitive changes consistent with CIND among whites, but more research is needed to understand the factors that influence scores among African Americans.

This study contains some potential limitations. For instance, the agreement between CERAD TS and clinical diagnoses may not be entirely unexpected, because these data were among the information that was considered in assigning clinical diagnoses in ADAMS. The IQCODE, on the other hand, was completed at the time of the study visit, but was not used in the diagnostic process. With respect to CERAD TS, we emphasize that: 1) the CERAD battery was presented as separate test components at the time of diagnosis, with no reference to the CERAD TS, which was calculated solely for the purposes of the present study; and 2) the CERAD battery was one element of a more extensive neuropsychological examination that included additional assessments of 
Table 2

Multinomial logistic regression of CIND and dementia on CERAD TS, with covariates, and stratified by race

\begin{tabular}{lllrll}
\hline & COR & \multicolumn{1}{l}{ SE } & \multicolumn{1}{c}{$t$} & $P$ value & $95 \%$ CI \\
\hline African American & & & & & \\
N vs. CIND & & & & & \\
$\quad$ Age & 1.072 & 0.072 & 1.04 & 0.311 & $0.933-1.231$ \\
$\quad$ Sex & 1.311 & 1.239 & 0.29 & 0.777 & $0.184-9.358$ \\
Education & 1.073 & 0.177 & 0.43 & 0.672 & $0.762-1.512$ \\
$\quad$ CERAD TS & 0.832 & 0.042 & -3.65 & 0.002 & $0.750-0.924$ \\
N vs. D & & & & & \\
$\quad$ Age & 0.978 & 0.115 & -0.19 & 0.854 & $0.766-1.249$ \\
Sex & 1.213 & 1.440 & 0.16 & 0.872 & $0.103-14.323$ \\
Education & 1.411 & 0.322 & 1.51 & 0.146 & $0.878-2.267$ \\
$\quad$ CERAD TS & 0.657 & 0.064 & -4.34 & 0.000 & $0.537-0.804$ \\
White & & & & & \\
N vs. CIND & & & & & \\
$\quad$ Age & 1.059 & 0.033 & 1.85 & 0.075 & $0.994-1.130$ \\
Sex & 1.129 & 0.330 & 0.41 & 0.682 & $0.618-2.060$ \\
Education & 1.085 & 0.065 & 1.37 & 0.183 & $0.960-1.226$ \\
CERAD TS & 0.834 & 0.020 & -7.64 & 0.000 & $0.795-0.876$ \\
N vs. D & & & & & \\
$\quad$ Age & 1.056 & 0.052 & 1.11 & 0.275 & $0.955-1.169$ \\
$\quad$ Sex & 1.234 & 0.558 & 0.47 & 0.645 & $0.488-3.124$ \\
Education & 1.195 & 0.092 & 2.33 & 0.028 & $1.021-1.399$ \\
CERAD TS & 0.681 & 0.024 & -10.98 & 0.000 & $0.634-0.732$ \\
\hline
\end{tabular}

Abbreviations: CERAD TS, Consortium to Establish a Registry for Alzheimer's Disease neuropsychological battery, total score; N, normal base value for models; CIND, cognitive impairment, not demented; D, dementia; COR, conditional odds ratio; SE, standard error; $95 \% \mathrm{CI}, 95 \%$ confidence interval.

Age was modeled in years, education was modeled as years obtained, and sex was modeled as female $=1$.

memory, attention, language, and executive function. In addition, the neuropsychological examination was one aspect of a comprehensive clinical assessment that included a clinical and medical history, other informant reports of cognition and functional performance, applicable medical records, and a brief neurologic examination. The totality of this information was used by a consensus panel of psychologists and physicians, who judged these data with respect to formal diagnostic criteria and from the perspective of their own specializations and clinical experience. Thus, although components of the CERAD TS performance were known in some form at the time of diagnosis, it is unlikely that the CERAD TS was overly influential in the diagnostic process.

We also note a correlation among some of the predictor variables, which may have accounted for unexpected trends of increased odds of cognitive impairment in association with higher level of education in models with the CERAD TS. This finding is not consistent with our univariate results, and is likely attributable to the influence of educational level on CERAD TS. Correlations between educational level and neuropsychological tests are difficult to avoid, which highlights a need for neurocognitive tests that are sensitive to cognitive impairment, while decreasing the influence of education on test outcomes.

An important issue remains in terms of the generalization of our results. The sample size available to examine the risk
Table 3

Multinomial logistic regression of CIND and dementia on IQCODE, with covariates, and stratified by race

\begin{tabular}{lllrll}
\hline & COR & SE & \multicolumn{1}{c}{$t$} & $P$ value & $95 \%$ CI \\
\hline African American & & & & & \\
N vs. CIND & & & & & \\
$\quad$ Age & 1.052 & 0.076 & 0.70 & 0.490 & $0.905-1.222$ \\
$\quad$ Sex & 1.388 & 1.463 & 0.31 & 0.759 & $0.155-12.433$ \\
Education & 0.813 & 0.086 & -1.96 & 0.064 & $0.653-1.013$ \\
$\quad$ IQCODE & 1.027 & 0.026 & 1.05 & 0.306 & $0.974-1.08$ \\
N vs. D & & & & & \\
$\quad$ Age & 1.138 & 0.102 & 1.43 & 0.166 & $0.944-1.372$ \\
Sex & 1.474 & 0.818 & 0.70 & 0.493 & $0.464-4.676$ \\
Education & 0.805 & 0.108 & -1.62 & 0.120 & $0.609-1.063$ \\
IQCODE & 1.152 & 0.061 & 2.67 & 0.014 & $1.031-1.286$ \\
White & & & & & \\
N vs. CIND & & & & & \\
$\quad$ Age & 1.120 & 0.033 & 3.82 & 0.001 & $1.054-1.190$ \\
Sex & 0.621 & 0.157 & -1.88 & 0.071 & $0.369-1.045$ \\
Education & 0.882 & 0.037 & -2.97 & 0.006 & $0.809-0.962$ \\
$\quad$ IQCODE & 1.077 & 0.019 & 4.32 & 0.001 & $1.039-1.116$ \\
N vs. D & & & & & \\
$\quad$ Age & 1.148 & 0.055 & 2.89 & 0.008 & $1.041-1.266$ \\
Sex & 1.032 & 0.430 & 0.08 & 0.941 & $0.438-2.430$ \\
Education & 0.811 & 0.049 & -3.49 & 0.002 & $0.717-0.917$ \\
IQCODE & 1.162 & 0.023 & 7.52 & 0.000 & $1.115-1.211$ \\
\hline
\end{tabular}

Abbreviations: IQCODE, Informant Questionnaire of Cognitive Status in the Elderly; N, normal base value for models; CIND, cognitive impairment, not demented; D, dementia; COR, conditional odds ratio; SE, standard error; 95\% CI, 95\% confidence interval.

Age was modeled in years, education was modeled as years obtained, and sex was modeled as female $=1$.

of cognitive impairment among African Americans was small for models with three diagnostic groups and three covariates. It is possible that with a larger sample size, we might have detected a significant association of IQCODE with predicting CIND among African Americans. With a larger sample size, we may also have been able to explore the trend of a decreased likelihood of CIND among white women in models with IQCODE, which may reflect an interesting interaction of both race and sex in informant reports of cognitive function. We also lacked the sample size to evaluate subtypes of CIND and dementia, which are heterogeneous in terms of clinical course and presentation, and which may have had different individual associations with CERAD TS and IQCODE. Only a few population-based studies in the research literature had a large enough sample size to examine subtypes of CIND and dementia diagnoses among African Americans relative to whites or other racial and ethnic groups in the United States, and many of these studies were regionally and culturally specific. This highlights the need for population-based studies of cognitive impairment and dementia with sufficient samples sizes of nonwhite populations, but the greatest utility would arise from a study on a nationally representative scale, using a single methodology of assessment and diagnosis. Such a study might not only promote an understanding of the questions raised by the present study, but might also examine the contributions of multiple 
demographic, cultural, and medical influences that are important in understanding racial and ethnic disparities in outcomes of cognitive impairment and dementia. A better understanding of racial and ethnic disparities in cognitive impairment, dementia, and other age-related medical conditions will be needed to effectively meet the needs of the aging, diverse United States population.

The present study found that CERAD TS identified dementia and CIND in both whites and African Americans, but that IQCODE provided divergent results between whites and African Americans when cognitive impairment was below the threshold of dementia. Additional research is needed to understand how cultural perceptions may influence the perception of cognitive function when the presentation is emergent or heterogeneous in nature.

\section{Acknowledgments}

The National Institute on Aging provided funding for the Health and Retirement Study and the Aging, Demographics, and Memory Study (U01 AG09740), data from which were used in this analysis. This study was also supported by National Institute on Aging grant R01 AG027010. We gratefully acknowledge the scientific and clinical input of the other members of the consensus conference panel: Kathleen A. Welsh-Bohmer, PhD, John C.S. Breitner, MD, Norman L. Foster, MD, Bruno Giordani, PhD, Hugh Hendrie, MB, $\mathrm{ChB}$, DSc, and Frederick W. Unverzagt, PhD. In addition, Drs. Breitner and Welsh-Bohmer were involved in the early planning and development of the ADAMS project, and in the efforts to obtain funding for the project. We also acknowledge the invaluable contributions of the ADAMS participants and the research staff at Duke University Medical Center and the University of Michigan.

\section{References}

[1] U.S. Census Bureau. Table 1. Annual estimates of the population by five-year age groups and sex for the United States: April 1, 2000 to July 1, 2006. Available at: http://www.census.gov/popest/national/ asrh/NC-EST2006-sa.html. Accessed December 16, 2008.

[2] Tang MX, Cross P, Andrews H, Jacobs DM, Small S, Bell K, et al. Incidence of AD in African-Americans, Caribbean Hispanics, and Caucasians in northern Manhattan. Neurology 2001;56:49-56.

[3] Demirovic J, Prineas R, Loewenstein D, Bean J, Duara R, Sevush S, et al. Prevalence of dementia in three ethnic groups: the South Florida Program on Aging and Health. Ann Epidemiol 2003;13:472-8.

[4] Fitzpatrick AL, Kuller LH, Ives DG, Lopez OL, Jagust W, Breitner JCS, et al. Incidence and prevalence of dementia in the Cardiovascular Health Study. J Am Geriatric Soc 2004;52:195-204.

[5] Plassman BL, Langa KM, Fisher GG, Heeringa SG, Weir DR, Ofstedal MB, et al. The prevalence of dementia in the United States: the Aging, Demographics, and Memory Study. Neuroepidemiology 2007;29:125-32.

[6] Lopez OL, Jagust WJ, Dulberg C, Becker JT, DeKosky ST, Fitzpatrick A, et al. Risk factors for mild cognitive impairment in the Cardiovascular Health Study Cognition Study. Arch Neurol 2003; 60:1394-9.
[7] Manly JJ. Cultural issues. In: Attix DK, Welsh-Bohmer KA, eds. Geriatric Neuropsychology: Assessment and Intervention. New York: Guilford Press; 2006. p. 198-222.

[8] McDougall GJ Jr, Vaughan PW, Acee TW, Becker H. Memory performance and mild cognitive impairment in black and white community elders. Ethn Dis 2007;17:381-8.

[9] Bohnstedt M, Fox PJ, Kohatsu ND. Correlates of Mini-Mental Status Examination scores among elderly demented patients. The influence of race-ethnicity. J Clin Epidemiol 1994;47:1381-7.

[10] Welsh KA, Fillenbaum G, Wilkinson W, Heyman A, Mohs RC, Stern Y, et al. Neuropsychological test performance in African-American and white patients with Alzheimer's disease. Neurology 1995; 45:2207-11.

[11] Fillenbaum GG, Heyman A, Huber MS, Ganguli M, Unverzagt FW. Performance of elderly African American and white community residents on the CERAD Neuropsychological Battery. J Int Neuropsychol Soc 2001;7:502-9.

[12] Manly JJ, Jacobs DM, Sano M, Bell K, Merchant CA, Small SA, et al. Cognitive test performance among nondemented elderly African Americans and whites. Neurology 1998;50:1238-45.

[13] Burns R, Nichols LO, Graney MJ, Martindale-Adams J, Lummus A. Cognitive abilities of Alzheimer's patients: perceptions of black and white caregivers. Int J Aging Hum Dev 2006;62:209-19.

[14] Jorm AF, Jacomb PA. The Informant Questionnaire on Cognitive Decline in the Elderly (IQCODE): socio-demographic correlates, reliability, validity and some norms. Psychol Med 1989;19: 1015-22.

[15] Morales JM, Gonzalez-Montalvo JI, Bermejo F, Del-Ser T. The screening of mild dementia with a shortened Spanish version of the "Informant Questionnaire on Cognitive Decline in the Elderly." Alzheimer Dis Assoc Disord 1995;9:105-11.

[16] Fuh JL, Teng EL, Lin KN, Larson EB, Wang SJ, Liu CY, et al. The Informant Questionniare on Cognitive Decline in the Elderly (IQCODE) as a screening tool for dementia for a predominantly illiterate Chinese population. Neurology 1995;45:92-6.

[17] Narasimhalu K, Lee J, Auchus AP, Chen CP. Improving detection of dementia in Asian patients with low education: combining the Mini-Mental State Examination and the Informant Questionnaire on Cognitive Decline in the Elderly. Dement Geriatr Cogn Disord 2008; 25:17-22.

[18] Juster FT, Suzman R. An overview of the Health and Retirement Study. J Hum Resources 1995;30(Suppl):135-45.

[19] Willis RJ. Theory confronts data: how the HRS is shaped by the economics of aging and how the economics of aging will be shaped by the HRS. Labour Econ 1999;6:119-45.

[20] Soldo BJ, Hurd MD, Rodgers WL, Wallace RB. Asset and health dynamics among the oldest old: an overview of the AHEAD Study. J Gerontol [B] Psychol Sci Soc Sci 1997;52B:1-20.

[21] Ofstedal MB, Fisher G, Herzog AR. Documentation of cognitive functioning measures in the Health and Retirement Study. Available at: http:// hrsonline.isr.umich.edu/docs/userg/dr-006.pdf. Accessed December 16, 2008.

[22] Heeringa SG, Fisher GG, Hurd MD, Langa KM, Ofstedal MB, Plassman BL, et al. Aging, Demographics and Memory Study (ADAMS). Sample design, weights, and analysis for ADAMS. Available at: http://hrsonline.isr.umich.edu/meta/adams/desc/ADAMSSampleWeig hts_Nov2007.pdf. Accessed December 16, 2008.

[23] Langa KM, Plassman BL, Wallace RB, Herzog AR, Heeringa SG, Ofstedal MB, et al. The Aging, Demographics, and Memory Study: study design and methods. Neuroepidemiology 2005;25:181-91.

[24] Little RJA, Rubin DB. Statistical Analysis with Missing Data. 2nd ed. New York: John Wiley and Sons; 2002.

[25] Health and Retirement Study. Available at: http://hrsonline.isr.umich. edu/. Accessed December 16, 2008.

[26] Plassman BL, Steffens DC, Burke JR, Welsh-Bohmer KA, Newman TN, Drosdick D, et al. Duke Twins Study of Memory in Aging in the NAS-NRC Twin Registry. Twin Res Hum Genet 2006;9:950-7. 
[27] Plassman BL, Havlik RJ, Steffens DC, Helms MJ, Newman TN, Drosdick D, et al. Documented head injury in early adulthood and risk of Alzheimer's disease and other dementias. Neurology 2000; 55:1158-66.

[28] Tschanz JT, Welsh-Bohmer KA, Skoog I, West N, Norton MC, Wyse BW, et al. Dementia diagnoses from clinical and neuropsychological data compared. Neurology 2000;54:1290-6.

[29] Plassman BL, Khachaturian AS, Townsend JJ, Ball MJ, Steffens DC, Leslie CE, et al. Comparison of clinical and neuropathological diagnoses of AD in three epidemiological samples. Alzheimers Dement 2006; $2: 2-11$.

[30] American Psychiatric Association. Diagnostic and Statistical Manual of Mental Disorders. Third Edition-Revised. Washington, DC: American Psychiatric Association; 1987.

[31] American Psychiatric Association. Diagnostic and Statistical Manual of Mental Disorders. Fourth Edition. Washington, DC: American Psychiatric Association; 1994.

[32] McKhann G, Drachman D, Folstein M, Katzman R, Price D, Stadlan EM. Clinical diagnosis of Alzheimer's disease: report of the NINCDS-ADRDA Work Group under the auspices of the Department of Health and Human Services Task Force on Alzheimer's Disease. Neurology 1984;34:939-44.

[33] Roman GC, Tatemichi TK, Erkinjuntti T, Cummings JL, Masdeu JC, Garcia JH, et al. Vascular dementia: diagnostic criteria for research studies. Report of the NINDS-AIREN International Workshop. Neurology 1993;43:250-60.

[34] Lund and Manchester Groups Clinical and neuropathological criteria for frontotemporal dementia. J Neurol Neurosurg Psychiatry 1994; $57: 416-8$.

[35] McKeith IG, Galasko D, Kosaka K, Perry EK, Dickson DW, Hansen LA, et al. Consensus guidelines for the clinical and pathologic diagnosis of dementia with Lewy bodies (DLB): report of the Consortium on DLB International Workshop. Neurology 1996;47:1113-24.

[36] Breitner JCS, Wyse BW, Anthony JC, Welsh-Bohmer KA, Steffens DC, Norton MC, et al. APOE-epsilon4 count predicts age when prevalence of $\mathrm{AD}$ increases, then declines: the Cache County Study. Neurology 1999;53:321-31.
[37] Plassman BL, Langa KM, Fisher GG, Heeringa SG, Weir DR, Ofstedal MB, et al. Prevalence of cognitive impairment without dementia in the United States. Ann Intern Med 2008;148:427-34.

[38] Morris JC, Heyman A, Mohs RC, Hughes JP, van Belle G, Fillenbaum G, et al. The Consortium to Establish a Registry for Alzheimer's Disease (CERAD). Part I. Clinical and neuropsychological assessment of Alzheimer's disease. Neurology 1989;39:1159-65.

[39] Chandler MJ, Lacritz LH, Hynan LS, Barnard HD, Allen G, Deschner M, et al. A total score for the CERAD neuropsychological battery. Neurology 2005;65:102-6.

[40] Jorm AF. A short form of the Informant Questionnaire on Cognitive Decline in the Elderly (IQCODE): development and cross-validation. Psychol Med 1994;24:145-53.

[41] Jorm AF. The Informant Questionnaire on Cognitive Decline in the Elderly (IQCODE): a review. Int Psychogeriatr 2004;16:275-93.

[42] StataCorp. Stata Statistical Software. Release 9 [program]. College Station, TX: StataCorp; 2005.

[43] StataCorp. Stata Technical Bulletin. College Station, TX: StataCorp; 2000.

[44] Miller B, Campbell RT, Farran CJ, Kaufman JE, Davis L. Race, control, mastery, and caregiver distress. J Gerontol [B] Psychol Sci Soc Sci 1995;50(Suppl):S374-82.

[45] Haley WE, West CA, Wadley VG, Ford GR, White FA, Barrett JJ, et al. Psychological, social, and health impact of caregiving: a comparison of black and white dementia family caregivers and noncaregivers. Psychol Aging 1995;10:540-52.

[46] Farran CJ, Miller BH, Kaufman JE, Davis L. Race, finding meaning, and caregiver distress. J Aging Health 1997;9:316-33.

[47] Gonzalez EW. Resourcefulness, appraisals, and coping efforts of family caregivers. Issues Ment Health Nurs 1997;18:209-27.

[48] Haley WE, Roth DL, Coleton MI, Ford GR, West CA, Collins RP, et al. Appraisal, coping, and social support as mediators of well-being in black and white family caregivers of patients with Alzheimer's disease. J Consult Clin Psychol 1996;64:121-9.

[49] Young RF, Kahana E. The context of caregiving and well-being outcomes among African and Caucasian Americans. Gerontologist $1995 ; 35: 225-32$. 